\title{
Faktoren der User Experience
}

\section{Systematische Übersicht über produktrelevante UX-Qualitätsaspekte}

\author{
Dominique Winter \\ Buhl Data Service $\mathrm{GmbH}$ \\ Am Siebertsweiher 3/5 \\ 57290 Neunkirchen \\ dwinter@buhl-data.com
}

Jörg Thomaschewski

Hochschule Emden/Leer

Constantiaplatz 4

26723 Emden

joerg.thomaschewski@hs-emden-leer.de

\author{
Martin Schrepp \\ SAP SE \\ Dietmar-Hopp-Allee 16 \\ 69190, Walldorf \\ martin.schrepp@sap.com
}

\begin{abstract}
User Experience ist eine Aggregation vieler unterschiedlicher Faktoren bzw. Qualitätsaspekte. Je nach Produkt sind andere Faktoren wichtig, um ein positives Nutzungserlebnis zu erzeugen. Für den Praktiker ist ein klares Bild der vorhandenen Faktoren wichtig, um für den Designprozess schon frühzeitig Schwerpunkte zu setzen (welche Faktoren sind für dieses Produkt wichtig). Auch helfen die Faktoren nach Fertigstellung des Produkts, die richtigen Fragebögen zur Evaluation der erreichten User Experience auszuwählen. Die für die Wahrnehmung der User Experience wichtigsten Qualitätsaspekte wurden durch Literaturanalyse, Expertenbefragungen und -diskussionen gesammelt und kritisch betrachtet. Dazu wurde eine erste Studie durchgeführt, um zu erfahren, welche Faktoren für welche Produkttypen besonders relevant sind. Es wird weiterhin für die verbreitetsten UX-Fragebögen eine Übersicht gegeben, welche der vorgestellten Faktoren durch den Fragebogen jeweils erfasst werden.
\end{abstract}

\section{Keywords}

User Experience, Fragebögen, UX-Qualitätsaspekte, UX-Faktoren, UX-Evaluation

\section{Einleitung}

Vergleicht man gängige Fragebögen zur User Experience, so stellt man fest, dass diese oft sehr unterschiedliche Faktoren bzw. Qualitätsaspekte der User Experience messen. Faktoren sind Einflussgrößen die das Gesamtkonstrukt User Experience entweder repräsentieren oder 
signifikant beeinflussen. In relevanten wissenschaftlichen Artikeln werden sehr unterschiedliche Faktoren untersucht, von denen man annimmt, dass sie die User Experience beeinflussen oder bestimmen. Allein die ISO 9241-210 nennt über 20 solcher Einflussfaktoren. Einige dieser Faktoren liegen im Nutzer (z.B. aktuelle Gemütslage), aber viele andere lassen sich erst durch die Interaktion mit dem Produkt fassen (z.B. Steuerbarkeit).

Eine weitere Schwierigkeit liegt darin, dass manche Faktoren nur für bestimmte Produkte relevant sind bzw. die Wichtigkeit dieser Faktoren für die Wahrnehmung der User Experience zwischen verschiedenen Produktkategorien stark variiert. Für eine Anwendung zur Erfassung der Steuererklärung spielt das wahrgenommene Vertrauen in Datensicherheit und Vertraulichkeit eine andere Rolle als bei einem Web-Radio. Für einen Self-Service im Internet, z.B. das Rückmelden von Zählerständen an einen Versorger, ist intuitive Bedienung von zentraler Bedeutung. Für eine von Experten häufig genutzte betriebswirtschaftliche Anwendung spielt dieser Faktor eher eine untergeordnete Rolle. Hier sind andere Faktoren (z.B. Leichtigkeit) deutlich wichtiger.

Wir geben in diesem Beitrag einen Überblick der wichtigsten, für die User Experience relevanten Faktoren, die durch Literaturanalyse, Expertenbefragungen und -diskussionen gesammelt und kritisch betrachtet wurden. Zusätzlich werden wir anhand der Daten einer ersten Studie diskutieren, welche Qualitätsaspekte für welche Produkttypen besonders relevant sind. Die Faktoren wurden in einer Umfrage zur Beurteilung von weitverbreiteten Produkten wie MS Word oder WhatsApp getestet. Dazu mussten die Teilnehmer beantworten, wie sehr der jeweilige Faktor ihrer Meinung nach auf dieses Produkt zutrifft und wie wichtig dieser Faktor bei solchen Produkten ist.

Für den Praktiker ist ein klares Bild der vorhandenen UX-relevanten Faktoren einerseits wichtig, um für den Designprozess schon frühzeitig Schwerpunkte zu setzen (welche Qualitätsaspekte sind für dieses Produkt wichtig). Andererseits helfen sie auch nach Fertigstellung des Produkts die richtigen Fragebögen und Fragestellungen zur Evaluation der User Experience auszuwählen.

\section{Faktoren der User Experience}

Wir geben im Folgenden eine Übersicht der aus unserer Sicht wichtigsten UX-Faktoren, die durch Literaturanalyse, Expertenbefragungen und -diskussionen zusammengetragen wurden. Einige Faktoren werden in der Literatur unter einer alternativen Bezeichnung genannt. In diesem Fall stehen die alternativen Bezeichnungen in Klammern hinter dem in diesem Beitrag verwendeten Namen bzw. wir geben diesen bei den Quellen explizit mit an.

Fast alle Faktoren tauchen in einer Vielzahl von Quellen und Publikationen auf. Das Auflisten aller Quellen zu den verschiedenen Faktoren würde den Rahmen dieses Beitrags sprengen, deshalb wurden nur die unserer Ansicht nach relevantesten Quellen angegeben. Ist keine Quelle angegeben, stammt der Faktor aus Expertenbefragungen und -diskussionen, an der neben den drei Autoren zwei weitere Personen mit umfangreichen Erfahrungen in der 
Produktentwicklung teilgenommen haben. Bei diesen Expertenbefragungen und diskussionen wurden zuerst mögliche Faktoren gesammelt und anschließend anhand verschiedener Produktszenarien potentielle Faktoren ermittelt. Hierbei wurden einige neue Faktoren erhalten, die sich noch nicht in vorhandenen Fragebögen finden.

Falls es einen Fragebogen gibt, der eine entsprechende Skala enthält, wird dieser angegeben. Skalen bestehen aus mehreren inhaltlich zusammenhängenden Fragen, die gemeinsam ausgewertet werden, um ein Messergebnis zu ermitteln. Auch hier konnten natürlich nicht alle vorhandenen Fragebögen berücksichtigt werden. Wir haben uns auf die folgenden relativ häufig verwendeten Fragebögen beschränkt: IsoMetrics (Gediga \& Hamborg 1999), ISONORM (Prümper 1997), SUMI (Kirakowski 1996), UEQ (Laugwitz et al. 2008), AttrakDiff (Hassenzahl et al. 2003), meCUE (Minge \& Riedel 2013), VISAVIS (Thielsch \& Moshagen 2011) und SUPRQ (Sauro, 2015).

Fragebögen, die nur eine globale UX-Dimension messen, d.h. keine klar abgegrenzten Faktoren aufweisen, z.B. der SUS (Brooke 1986) wurden nicht berücksichtigt.

Aktualität (Inhaltsqualität, Content-Qualität): Die Informationen, die das Produkt dem Nutzer liefert, sind stets auf dem aktuellen Stand und von guter Qualität. Bei Webseiten ist die Aktualität gegeben, wenn die Informationen in der Seite dem aktuellen Wissens- bzw. Datenstand entsprechen. In einer betriebswirtschaftlichen Finanzanwendung, wenn diese die aktuellen gesetzlichen Regelungen implementiert. Quelle: Bargas-Avila et al. (2009), Laugwitz et al. (2009). Der Intranet Satisfaction Questionnaire (Bargas-Avila et al 2009) enthält eine Skale zum Messung der Qualität der Information auf Intranet-Seiten. Eine zusätzliche Skala zum UEQ Fragebogen bei dessen Anwendung innerhalb der DATEV AG erfragt, ob eine betriebswirtschaftliche Anwendung die aktuellen gesetzlichen Regelungen implementiert. Im SUPRQ ist eine Skala Credibility enthalten, die Aspekte von Aktualität und Vertrauen in einer Skala vereinigt.

Anpassbarkeit (Individualisierbarkeit, Personalisierbarkeit): Der Nutzer kann das Produkt an seine persönlichen Bedürfnisse und Anforderungen bzw. seinen Arbeitsstil anpassen. In einem professionell genutzten Produkt kann dies zum Beispiel durch das Ausblenden nicht genutzter Felder oder Funktionen erfolgen. In einem sozialen Netzwerk durch die Möglichkeit Einstellungen dafür vorzunehmen, für wen welche Informationen sichtbar gemacht werden. Quelle: ISO 9241 (Teil 110 - Individualisierbarkeit). Skala im ISONORM und IsoMetrics Fragebogen.

Bequemlichkeit: Das Produkt erleichtert das Leben. Dies wird als ein von der Nützlichkeit abgegrenzter Faktor angesehen, da ein zusätzlicher Nutzen auch Möglichkeiten bietet, die sonst nicht vorhanden sind, d.h. nicht unbedingt in einer Erleichterung des Lebens münden muss.

Durchschaubarkeit (Erlernbarkeit, Lernförderlichkeit): Ist es einfach, die Bedienung des Produkts $\mathrm{zu}$ verstehen und $\mathrm{zu}$ erlernen? Quelle: ISO 9241 (Teil 110 - dort als Lernförderlichkeit bezeichnet). Als Skala im UEQ, SUMI, SUS, ISONORM und IsoMetrics vorhanden. 
Effizienz: Der Nutzer kann seine Ziele mit minimalem zeitlichem und physischem Aufwand erreichen. Das Produkt reagiert schnell auf Nutzereingaben. Das Design der Nutzerschnittstelle macht keine unnötigen Arbeitsschritte erforderlich. Quelle: ISO 9241 (Teil 11) bzw. (Teil 110 - Aufgabenangemessenheit). Zur Messung existieren eigene Skalen im UEQ und SUMI. In den Fragebögen ISONORM und IsoMetrics deckt die Skala Aufgabenangemessenheit diesen Faktor mit ab.

Identität: Ich kann mich mit dem Produkt identifizieren und nehme Eigenschaften des Produkts in mich auf. Quelle: Hassenzahl et al. (2003), Faktor Identität des AttrakDiff Fragebogens, Skala Status im meCUE.

Immersion: Wenn der Nutzer sich mit dem Produkt beschäftigt, vergisst dieser die Zeit. Der Nutzer kann völlig in der Beschäftigung mit dem Produkt versinken. Dieses Qualitätskriterium ist insbesondere bei Spielen und anderen zur Unterhaltung dienenden Produkten relevant.

Intuitive Bedienung: Kann der Nutzer die Benutzerschnittstelle mit seinen vorhandenen Fähigkeiten unmittelbar und ohne jegliche Einarbeitung oder Anleitung durch andere bedienen? Quelle: Mohs et al. (2006).

Leichtigkeit: Es ist für den Nutzer einfach seine Aufgaben mit dem Produkt zu erledigen. Die Interaktion mit dem Produkt ist weder körperlich noch mental anstrengend. Quelle: Hart \& Staveland (1988) NASA-TLX (Task Load Index).

Neuartigkeit: Das Produkt ist neu und innovativ. So etwas gab es bisher nicht.

Nützlichkeit: Die Benutzung des Produkts bringt Vorteile. Dies ist zum Beispiel der Fall, wenn das Produkt die Produktivität oder Effizienz bei betriebswirtschaftlichen Prozessen erhöht. Aber auch, wenn ein Self-Service im Web, dem Nutzer einen lästigen Behördengang erspart. Quelle: Davies (1989), Technology Acceptance Model, dort werden auch sechs Fragen zu Messung dieses Faktors angegeben. Auch der meCUE enthält eine Skala Nützlichkeit, die diesen Aspekt mit drei Fragen erfasst.

Originalität: Das Produkt ist interessant und ungewöhnlich gestaltet. Es erregt durch seine originelle Gestaltung das Interesse des Nutzers. Quelle: Laugwitz et al. (2008). Als Skala im UEQ vorhanden.

Schönheit (Ästhetik): Das Produkt ist schön und ansprechend gestaltet. Das Produkt macht auf den Nutzer einen ästhetischen Eindruck. Quellen: Moshagen \& Thielsch (2010) VisAWI Fragebogen, dieser liefert vier Unterfaktoren (Einfachheit, Vielseitigkeit, Farbigkeit, Kunstfertigkeit) zu diesem Konstrukt; Fragebogen von Lavie \& Tractinsky (2004), der die zwei Unterfaktoren klassische und expressive Ästhetik nennt. Auch der meCUE und der SUPRQ (Skala Appearance) enthalten entsprechende Skalen.

Soziales: Die Beschäftigung mit dem Produkt hilft mir Kontakte zu knüpfen und mich selbst positiv darzustellen.

Spaß: Die Interaktion mit dem Produkt macht dem Nutzer Freude. Trotz der Nähe zur Stimulation ist Spaß ein eigener Faktor, da bei der Stimulation die Motivation des Nutzers im 
Vordergrund steht, dass Produkt immer wieder zu nutzen, was aber nicht zwingend daraus entstehen muss, dass die Nutzung des Produkts Spaß macht. Die Motivation kann auch aus durchaus anderen Faktoren resultieren, z.B. Möglichkeiten zur persönlichen Entwicklung oder andere Anreize. Quelle: Hatscher (2001).

Steuerbarkeit (Kontrollierbarkeit, Fehlertoleranz, Robustheit): Das Produkt reagiert immer vorhersehbar und konsistent auf Nutzerinteraktionen. Der Nutzer hat stets die volle Kontrolle über das Produkt. Das Produkt ist fehlertolerant. Quelle: ISO 9241 (Teil 110), dort wird dieser Aspekt in die beiden Teilaspekte Steuerbarkeit und Fehlertoleranz aufgespalten, die als unabhängige Teilaspekte behandelt werden. Als Skala im SUMI, UEQ, ISONORM und IsoMetrics vorhanden.

Stimulation: Die Arbeit mit dem Produkt motiviert den Nutzer immer wieder mit ihm zu arbeiten. Es ist anregend und spannend mit dem Produkt zu arbeiten. Quelle: Laugwitz et al. (2008). Skala des AttrakDiff und UEQ-Fragebogens.

Übersichtlichkeit (visuelle Komplexität): Die Benutzerschnittstelle wirkt aufgeräumt und übersichtlich und hat eine geringe visuelle Komplexität. Der Nutzer findet sich schnell darin zurecht. Quelle: Müller \& Schrepp (2013), dort wird auch eine einzelne Frage zur Messung beschrieben.

Verbundenheit (Loyalität, Bindung): Auch wenn es andere, bessere Produkte für die gleichen Aufgaben gibt, wird der Nutzer nicht wechseln. Nutzer fühlen sich durch die bisherigen positiven Erfahrungen mit dem Produkt, der Marke oder dem Anbieter verbunden. Kann durch die Skala Produktloyalität des meCUE Fragebogens erfasst werden (es gibt dort auch eine Skala Bindung mit ähnlichem Inhalt). Der SUPRQ enthält eine eigene Skala Loyalty mit zwei Fragen zur Messung dieses Aspekts.

Vertrauen: Die Daten und Informationen, die der Nutzer bei der Interaktion mit dem Produkt eingibt, sind in sicheren Händen. Dies spielt natürlich vor allem bei Produkten eine Rolle, bei denen der Nutzer kritische Daten preisgibt, z.B. beim Einkaufen in Web-Shops oder Online-Banking. Quelle: Corritore et al. (2001).

Vollständigkeit: Das Produkt bietet dem Nutzer alles, was er oder sie erwartet. Es fühlt sich vollständig an, auch wenn es nicht tatsächlich alles im konkreten Nutzungskontext Notwendige bieten sollte. Der Faktor Vollständigkeit bezieht sich somit mehr auf die wahrgenommene Vollständigkeit und weniger auf die Summe der aufgabenangemessenen Nutzungskontexte.

Wertigkeit (Professionelle Gestaltung, Kunstfertigkeit): Das Produkt macht einen hochwertigen und soliden Eindruck. Die Gestaltung des Produkts wirkt professionell. Quellen: Moshagen \& Thielsch (2010) - VisAWI Fragebogen, dieser liefert mit dem Unterfaktor Kunstfertigkeit einen Teilaspekt zur Wertigkeit. 


\section{Faktorenrelevanz für unterschiedliche Produkte}

Nicht jeder der genannten Qualitätsaspekte ist in allen Situationen relevant. Es hängt einerseits vom befragten Nutzer ab, wie wichtig ihm ein solcher Aspekt ist. Andererseits wird dies auch zwischen Produkten stark variieren, d.h. derselbe Nutzer wird einen dieser Aspekte bei einem Produkt wichtig finden und bei einem anderen nicht.

Für einige der Qualitätsaspekte ist naheliegend, dass sie nur bei bestimmten Produkten überhaupt sinnvoll sind. Bei Identität geht es z.B. darum, dass der Nutzer über den Besitz (z.B. ein Smartphone eines bestimmten Typs) oder die Benutzung eines Produkts etwas über sich kommuniziert und dadurch Kontakte knüpft oder sein Ansehen mehrt. Dies setzt in gewisser Weise voraus, dass der Nutzer über die Nutzung bzw. Nicht-Nutzung dieses Produkts selbst entscheidet oder entscheidend beitragen kann. Bei einer Business Software, die beruflich genutzt und von der Firma beschafft wird (d.h. der Nutzer hat hier in der Regel keine Entscheidung zu treffen), spielt dieser Aspekt eine untergeordnete Rolle.

In einer exemplarischen Studie wurden 56 Probanden in einer Onlineumfrage nach ihren Einschätzungen $\mathrm{zu}$ den vorgenannten, einzelnen Faktoren für Produkte aus den drei Produktkategorien Textverarbeitung, Kommunikation und Webbrowser befragt. Ziel der Umfrage war es zu erkennen, welche dieser Faktoren für die drei beispielhaften Produktkategorien von den Nutzern als wichtig eingestuft wurden und welche nicht.

Die Faktoren wurden in einer 7-stufigen Zustimmungsskala abgefragt, wobei für jeden Faktor die Aussage, dass dieser Faktor wichtig sei zu bewerten war (z.B. „Steuerbarkeit ist mir bei Produkten wie Firefox wichtig.“). Erkenntnisse aus einer solchen Umfrage könnten genutzt werden um die Faktorenrelevanz für eine Produktkategorie zu bestimmen. Wie auszugsweise in Abbildung 1 zu erkennen, können Aussagen aus einer solchen Befragung hergeleitet werden:

- Intuitive Bedienung, Nützlichkeit und Leichtigkeit sind über alle drei Produktkategorien von sehr hoher Wichtigkeit.

- Soziales ist für Textverarbeitungen nicht so wichtig wie für Kommunikationsanwendungen.

- Aktualität und Anpassbarkeit ist bei Browsern wichtiger als bei Textverarbeitungen und Kommunikationsprogrammen.

Auf Grundlage solcher Umfrageergebnisse könnte nun eine Auswahl von Fragebögen erfolgen, die vor allem die wichtigen Faktoren abfragt und unwichtige ausspart. Dadurch ist es möglich die Belastung der Probanden durch Vermeiden unnötiger Fragen gering zu halten und deren Beteiligung bzw. Bereitschaft zur Unterstützung zu erhöhen. 


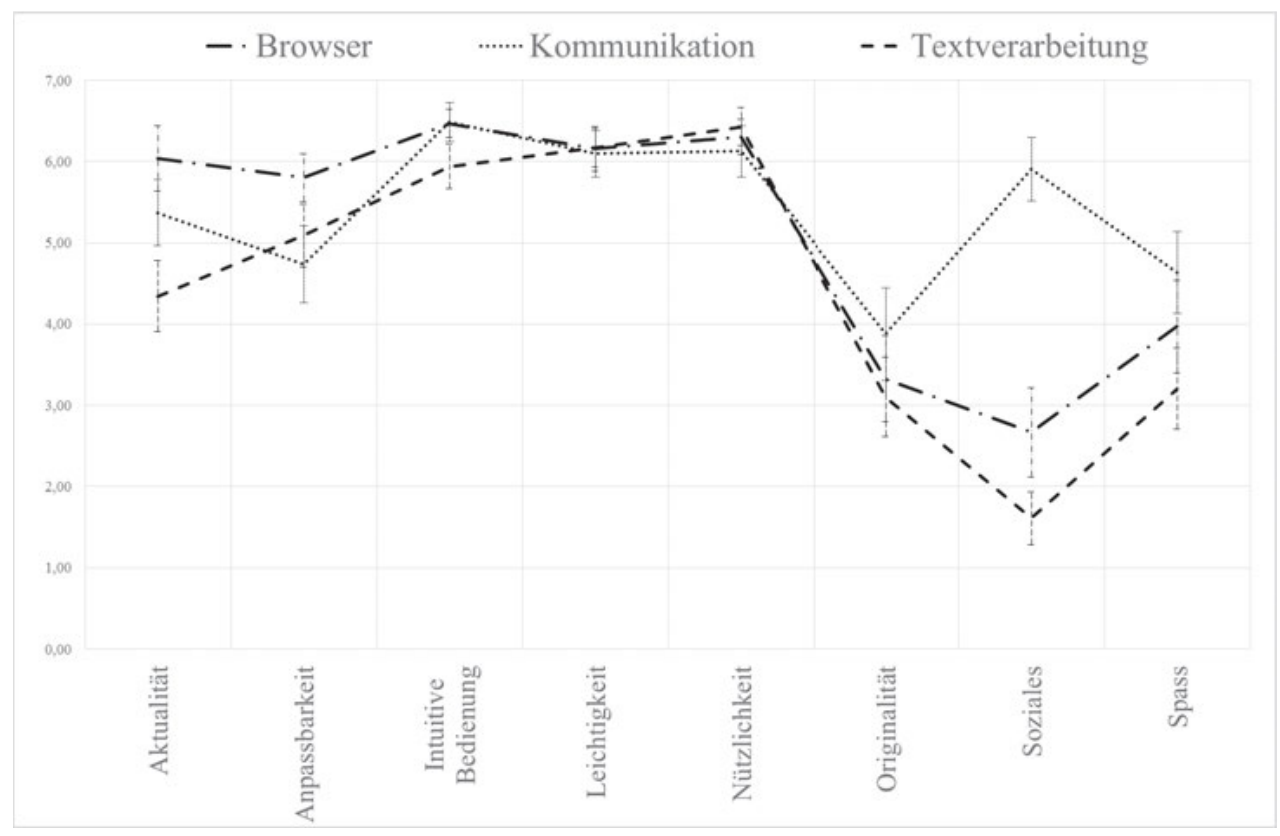

Abbildung 1: Faktorengewichtung durch Nutzerbewertung

\section{Zusammenfassung und Ausblick}

Wir haben eine Übersicht über relevante UX-Qualitätsaspekte gegeben, die auf das Gesamtkonstrukt UX einzahlen. Die Liste der angeführten Aspekte ist natürlich nicht vollständig. Einerseits gibt es immer neue Nutzungsszenarien, bei denen dann auch neue Aspekte zur Beurteilung der UX zum Tragen kommen können. Vor Einführung von WebShops oder Online-Banking musste man sich zum Beispiel über ein Kriterium wie Vertrauen weniger Gedanken machen. Dieses Kriterium tauchte erst mit der Verbreitung dieser neuen Möglichkeiten sehr prominent in der UX-Literatur auf. Andererseits gibt es sehr unterschiedlicher Produkte. Daher können für spezielle Anwendungen weitere Kriterien relevant sein, die in dieser Liste nicht genannt worden sind, aber stark auf die UX einwirken. Die in diesem Artikel dargestellte Liste aller relevanten UX-Faktoren wird sich entsprechend den zukünftigen Anwendungen und Nutzerbedürfnissen weiterentwickeln.

Der Vergleich der Qualitätsaspekte mit den wichtigsten UX-Fragebögen zeigt, dass keiner dieser Fragebögen in der Lage ist alle Aspekte abzudecken. Das ist wegen der Abhängigkeit der Art des Produkts und der damit verbundenen Zielgruppen natürlich auch nicht sinnvoll. Allerdings bedeutet dies auch, dass man ggfs. mehrere Fragebögen verwenden muss, um alle Aspekte abzudecken, die man für ein zu evaluierendes Produkt für wichtig erachtet. Da die verschiedenen Fragebögen in Bezug auf Messung und Darstellung der Resultate nicht einheitlich sind, ist das für die Datenerhebung nicht förderlich, da die befragten Nutzer innerhalb einer Befragung mit sehr verschiedenen Frageformaten konfrontiert werden. 
Naheliegend wäre es, auf Basis dieser Faktoren einen flexiblen und erweiterbaren Fragenkatalog zu entwickeln. Der UX-Professional könnte dann für jede Evaluation eines Produkts zuerst entscheiden, welche Qualitätsaspekte relevant sind und diese dann durch eine direkt in einen passgenauen Fragebogen überführen. Damit würden nur genau die als relevant angesehenen Aspekte erfragt. Wegen der inhärenten Unvollständigkeit solcher Kataloge, sollte das Fragenformat so gewählt sein, dass weitere Qualitätsaspekte leicht ergänzt werden können.

Man verliert bei einer solchen flexiblen Vorgehensweise jedoch Vorteile standardisierter Messinstrumente. Etablierte Fragebögen bieten oft einen Benchmark an, mit dem man sein Evaluationsergebnis mit dem einer großen Menge von bereits evaluierten Produkten vergleichen kann. Das Erstellen eines solchen Benchmarks wird bei passgenau zugeschnittenen Fragebögen schwieriger, da pro Skala eine geringere Menge an Evaluationen anfällt, aus denen man einen sinnvollen Benchmark berechnen kann.

\section{Literatur}

Bargas-Avila, J. A., Lötscher, J., Orsini, S. \& Opwis, K. (2009). Intranet satisfaction questionnaire: Development and validation of a questionnaire to measure user satisfaction with the intranet. Computers in Human Behavior, 25, 12411250.

Brooke, J. (1986). SUS: a quick and dirty usability scale. In Jordan, P.W., Thomas, B., Weerdmeester, B.A. \& McClelland, A.L. (Hrsg.): Usability Evaluation in Industry. London: Taylor and Francis.

Corritore, C.L., Wiedenbeck, S. \& Kracher, B. (2001). The elements of online trust. In CHI '01 Extended Abstracts on Human Factors in Computing Systems (CHI EA '01). ACM, New York, NY, USA, 504-505. DOI=10.1145/634067.634355 http://doi.acm.org/10.1145/634067.634355

Davis, D. (1989). Perceived usefulness, perceived ease of use, and user acceptance of information technology. MIS Quarterly, 13(3), 319339.

Deng, L. \& Poole, M. S. (2012). Aesthetic design of e-commerce web pages - Webpage Complexity, Order and preference. Electronic Commerce Research and Applications, 11(4), 420-440.

DIN EN ISO 9241-110 (2006). Ergonomics of human-system interaction - Part 110: Dialogue principles. Beuth, Berlin.

Gediga, G. \& Hamborg, K.C. (1999). IsoMetrics: Ein Verfahren zur Evaluation von Software nach ISO 9241/10. In Holling, H. \& Gediga, G. (Hrsg.): Evaluationsforschung, S. 195-234. Göttingen: Hogrefe.

Hart, S. \& Staveland, L. (1988). Development of NASA-TLX (Task Load Index): Results of empirical and theoretical research. In Hancock, P. \& Meshkati, N. (Hrsg.): Human mental workload (pp. 139-183). Amsterdam: North Holland.

Hassenzahl, M., Burmester, M. \& Koller, F. (2003). AttrakDiff: Ein Fragebogen zur Messung wahrgenommener hedonischer und pragmatischer Qualität. In Ziegler, J. \& Szwillus, G. (Hrsg.): Mensch \& Computer 2003. Interaktion in Bewegung. Stuttgart: Teubner. S. 187-196.

Hatscher, M. (2001). Joy of use - Determinanten der Freude bei der Software-Nutzung. In: Oberquelle, H., Oppermann, R. \& Krause, J. (Hrsg.), Mensch \& Computer 2001: 1. Fachübergreifende Konferenz. Stuttgart: B. G. Teubner. S. 445-446. 
Kirakowski, J. (1996). The Software Usability Measurement Inventory: Background and usage. In Jordan, P., Thomas B. \& Weerdmeester B. (Hrsg.), Usability Evaluation in Industry (S. 169- 178). London, UK: Taylor \& Francis.

Lavie, T. \& Tractinsky, N. (2004). Assessing dimensions of perceived visual aesthetics of web sites. International Journal of Human-Computer-Studies, 60, S. 269-298.

Laugwitz, B., Held, T. \& Schrepp, M. (2008). Construction and Evaluation of a User Experience Questionnaire. In Holzinger, A. (Hrsg.): HCI and Usability for Education and Work, LNCS 5298, Berlin, Heidelberg: Springer, S. 63-76.

Laugwitz, B., Schubert, U., Ilmberger, W., Tamm, N., Held, T. \& Schrepp, M. (2009). Subjektive Benutzerzufriedenheit quantitativ erfassen: Erfahrungen mit dem User Experience Questionnaire UEQ. In: Brau, H., Diefenbach, S., Hassenzahl, M., Kohler, F., Peissner, M., Petrovic, K., Thielsch, M., Ulrich, D. \& Zimmermann, D. (Hrsg.); Usability Professionals 2009, S. 220 - 225, Fraunhofer Verlag.

Minge, M. \& Riedel, L. (2013). meCUE - Ein modularer Fragebogen zur Erfassung des Nutzungserlebens. In Boll S., Maaß, S. \& Malaka, R. (Hrsg.): Mensch und Computer 2013: Interaktive Vielfalt (S. 89-98). München: Oldenbourg Verlag.

Mohs, C., Hurtienne, J., Israel, J. H., Naumann, A., Kindsmüller, M. C., Meyer, H.A. \& Pohlmeyer, A. (2006). IUUI - Intuitive Use of User Interfaces. In Bosenick, T., Hassenzahl, M., Müller-Prove, M. \& Peissner, M. (Hrsg.), Usability Professionals 06 (S. 130-133). Stuttgart: German Chapter der Usability Professionals' Association.

Moshagen, M. \& Thielsch, M. T. (2010). Facets of visual aesthetics. International Journal of HumanComputer Studies, 68, S. 689-709.

Müller, K. \& Schrepp, M. (2013). Visuelle Komplexität, Ästhetik und Usability von Benutzerschnittstellen. In Boll, S.; Maaß, S. \& Malaka, R. (Hrsg.), Mensch \& Computer 2013 Interaktive Vielfalt, S 211 - 220, München: Oldenbourg Verlag 2013.

Prümper, J. (1997). Der Benutzungsfragebogen ISONORM 9241/10: Ergebnisse zur Reliabilität und Validität. In Liskowski, R., Velichkovsky B.M. \& Wünschmann, W. (Hrsg.), Software-Ergonomie ' 97 - Usability Engineering: Integration von Mensch-Computer-Interaktion und SoftwareEntwicklung (pp. 253-262). Stuttgart: Teubner.

Sauro, J. (2015). SUPR-Q: A Comprehensive Measure of the Quality of the Website User Experience. In: Journal of Usability Studies 10 (2), S. 68-86.

Thielsch, M. T. \& Moshagen, M. (2011). Erfassung visueller Ästhetik mit dem VisAWI. In Brau, H., Lehmann, A., Petrovic, K. \& Schroeder, M.C. (Hrsg.), Usability Professionals 2011, S. 260-265. Stuttgart: German UPA e.V. 